\title{
INTERACTIVITY AND NON-LINEARITY: WHAT DO THEY REALLY MEAN?
}

\author{
Donald Marinelli \\ Professor of Drama \& Arts Management/ Co-Director, Entertainment Technology Center, \\ Carnegie Mellon UniversityPittsburgh, Pennsylvania
}

There is tremendous confusion regarding the terms interactivity and nonlinearity as they relate to digital media. My aspiration today is to tackle this subject head-on and hopefully clarify what these terms mean, the significance of each term, and how we should view these dynamics within the entertainment technology industry.

As a drama professor who resides now within computer science, much of my effort - and struggle - has been in creating a shared vocabulary between computer scientists, on the one hand, and artists, artisans, authors, designers, and craftspeople on the other. Some progress has been made, but frankly not enough. So, let us begin.

Interestingly enough, the terms interactivity and non-linearity are not to be found in my original profession. No playwright or screenwriter, for that matter, possesses nor uses those terms in his or her professional vocabulary. The reasoning for this is simple: any notion of interactivity in traditional storytelling media is intellectual and emotional; it is not physical. The quickest way to alienate a traditional storyteller is to charge that his or her medium is not interactive. The outraged reply will be that it is very interactive, thank you very much, but via the a psychological and physiological basis composed of attention, focus, consciousness, cognition, i.e., reasoning, reflection and emotional response (Aristotle's pity and fear, or perhaps the sentimental affirmation of a particular moral code, for example, bourgeoisie, Marxist, humanistic, religious, et al).

One might even hear protestations regarding the degree of physical involvement on the part of the traditional audience member. Doesn't fear,

The original version of this chapter was revised: The copyright line was incorrect. This has been corrected. The Erratum to this chapter is available at DOI: 10.1007/978-0-387-35660-0_65 
anger, empathy, identification, initiate some corresponding physical activity? Haven't you ever gripped the armrest of your chair due to terror, surprise, and shock? And yet, none of us here today would ever presume or declare that sitting in a seat watching a stage production is interactive, no matter how involved the viewer might appear to be. Interactivity, in our nomenclature, means something completely different. But what exactly does it mean?

Janet Murray in her now seminal work Hamlet on the Holodeck provided three measures of interactivity: immersion, agency, and transformation. While I hail Janet Murray for undertaking the first significant attempt at understanding the attributes of digital storytelling, I fear she errs on the side of scholarly nomenclature at the expense of a more readily available practical vocabulary. We underscore this by examining briefly Murray's first attribute: immersion.

No traditional-media storyteller worth his or her salt will concede immersion to digital media. From Coleridge's classic analysis of the "suspension of disbelief" to publicity-hype such as "edge-of-your-seat drama and adventure' the aspiration remains to have another human being leave his or her cares and woes by the door and enter into an imaginary world so 'real' that the viewer perceives and believes co-existence in time and space with the world being portrayed on stage or screen (glass or canvas). The best we can hope for here is to share in the 'aspiration' of immersion.

Digital media aspires to so affect another human being such that he or she will identify with the unfolding world or story at the expense of that same individual's mundane existence (and I use that term in a nonjudgmental way - mundane referring to a person's daily, ordinary existence, which for many people is fraught with daily drama and trauma). Immersion then is an umbilical chord we share with most storytelling intentionality.

What is distinct, though, is the degree of physical immersion brought about by digital media, from Omnimax theatres to head-mounted displays, from spatialized audio to haptic feedback devices, from olfactory stimulation to a third-person recording of our participation in a virtual world, technology effects the human corpus not only through affect, but also through multisensorial stimulation.

Janet Murray comes much closer to defining the distinction between traditional storytelling media and digital media when she focuses on the dynamic of agency. As a theatre person, I admit to being dubious about the term agency, primarily because my background is such that the word exists more often and more clearly as a noun than as a verb. The "agency" is, more often than not, where I go, rather than something I $d o$. Or, the agent (as in internal revenue agent, FBI agent or publicity agent) is whom I need to speak with concerning some important matter (a matter approached with fear 
and trepidation!). Yet it is in the verb form of the word that Murray begins to identify just what interactivity means.

Murray defines agency as the "satisfying power to take meaningful action and see the results of our decisions and choices." On the simplest level, we expect to feel agency on the computer when we double-click on a file and see it open before us. She rightfully observes that we do not usually expect to experience agency within a traditional narrative environment. Why not? Well, for a variety of reasons. More often than not we are sedentary, i.e., non-moving, seated or standing on terra firma. The primary reason though is because western storytelling is hierarchical enough in nature to reinforce the primacy of the storyteller over the listener.

An even more insidious reason can be found in the revolution of realism that occurred in late nineteenth century Europe. There is a direct connection between the establishment of psychology as the legitimate study of human behavior and a resulting creation of acting methodology. Western European culture was repressed enough to spawn an art form that replaced direct address, presentational and melodramatic acting styles with the "fourth wall." The "fourth wall" was the imaginary boundary that existed between the actors on stage and the audience seated in the orchestra and balcony. Audiences became voyeurs peering through this "fourth wall" onto the lives of the characters inhabiting the stage. And, like any good voyeur, you observed silently, keeping your thoughts, commentary, and actions to yourself. A sentimental tear was acceptable, as was a gasp in the proper place, all subtle indications to the cast that they were achieving their performance goals. Psychological realism, as it came to be called, has remained the predominant theatrical genre and performance style for over one hundred years.

That predominance has been under assault periodically usually through a resurgence of presentational acting in some instances, the Brechtian rebellion of the mid-twentieth century called "epic drama" in Eastern Europe, or attempts at reviving vaudeville, as with the "New Vaudevillians" of the 1980s. It wasn't until the advent of digital media and entertainment, however, and a generation of young people fed up with marginalization and the frustrating sense that their opinions - and votes - simply didn't matter, that the notion of interactive entertainment truly took hold. The truly revolutionary aspect of digital entertainment is that the audience member calls most of the shots (sometimes literally), and is not only welcomed to participate in the imaginary world, but is beckoned "on stage" and into the scene itself. Prior to this development, the closest traditional entertainment forms came to interactive entertainment was in participatory improvisation.

We comprehend the term agency as referring on one level to the dynamic of spatial navigation. Electronic environments offer the potential pleasure of 
spatial navigation, because the story is tied to the navigation of space. As I move forward, backwards, sideways, I feel a sense of powerfulness that is tied to my presence (and hopefully pleasure) in the unfolding story. My students and I have had heated discussions searching for a substitute term for agency, and empowerment surfaces frequently as a worthy challenger. Empowerment has merit due to the clear implication that the audience member now possesses the power and the means to intervene in a world outside of his or her own, an imaginary world brought to life by digital technology.

You cannot, however, endow an audience member with this capability, this significant attribute, and still consider him or her to be an "audience member." The transformation is elemental. Having the power to influence the world of others and their actions therein makes one at the very least an actor in the drama, at most an author determining the panoply of achievement within a world, as well as parameters of behavior (The SIMS is a terrific example of this). My biggest problem with a literary approach and critique to interactive storytelling has been use of the term narrative to define the world space. Digital media should be incorporating rightfully the language of live performance.

The nature of the digital beast is dramatic action, purposeful decision making within a defined objective that involves perceived physical movement through space. We can literally score a goal, touchdown, point, run, or wipe out an invader, destroy an alien spacecraft, capture an enemy strongpoint, win the court case, solve the murder. Aristotle is in fact alive and well in the foundational storytelling elements of plot, character, theme, diction, rhythm, and spectacle interwoven now with interactivity to form, essentially, a New Poetics.

The transformation is from noun to verb, audience member to actor. I point out to my students that standard police reports refer to the perpetrator of a crime as the actor. My publicity quip about the Entertainment Technology Center at Carnegie Mellon University is that "we turn computer science into a performing art." Digital storytellers need the insights of the Russian actor, director, and teacher, Konstantin Stanislavsky (or Michael Chekhov, or Lee Strasberg, or Uta Hagen, or...) as much or more so than literary theorists.

In digital entertainment, the study of behavior and motivation is as paramount as it is in the study of acting and drama. We can declare rightfully that within the realm of digital storytelling there no longer are "audience members" in the traditional meaning of the term, but rather, everyone becomes a participant. Improvisational acting is a required course in the Master of Entertainment Technology curriculum for the following reasons: 1) everyone partakes of meaningful action in an unfolding story; 2) 
improvisation succeeds only by making the other actor look good, thus underscoring the benefits of collaboration); and 3) improvisational acting comes closest to capturing the non-linearity made possible by digital storytelling.

Who could ask for a better transition to a discussion about non-linearity?

The difficulty in the term non-linearity is that it presumes an understanding of linearity, while both derivations presume a command of story and plot structure. This is quite a presumption. As a professor of drama for over 20 years, understanding plot structure, let alone knowing how to construct different plots, remains one of the most elusive goals in theatre, film, and television education.

Linearity and non-linearity has everything to do with plot. A story that runs its course is linear. A story that can only run its course is linear. Linearity is considered to be an absolute necessity by most traditional storytellers in order for the thematic intention of the story to be conveyed. Linearity is related to context. The author can never know with certainty the point-of-view (POV) of his or her audience, but certain presumptions can be made based upon genre, history, demographics, marketing, etc., for an author to have solid footing regarding the POV of the audience. To cite Janet Murray again: "A linear story, no matter how complex, moves towards a single encompassing version of a complex human event. Even those multiform stories that offer multiple retellings of the same event often resolve into a single "true" version - the viewpoint of the uninvolved eyewitness or the actual reality the protagonists wind up in after the alternate realities have collapsed."

Transforming a viewer into an active participant capable of making decisions affecting the story empowers the actor with an authority most traditional authors are unwilling to relinquish - and they don't know how regardless.

The revolution here is that non-linearity turns any 'story' into a plural: stories. Janet Murray captures the aspiration of everyone in this room when she says, "The challenge is in. creating stories that are goal driven enough to guide navigation but open-ended enough to allow free exploration and that display a satisfying dramatic structure no matter how the interactor chooses to traverse the space." Digital narratives add another powerful element by offering us the opportunity to enact stories rather then merely witness them. By enactment Janet Murray means an experience wherein 'interactors' (her term) are invited to enact or construct their own stories out of a set of formulaic elements. Enacted events have a transformative power that exceeds both narrated and conventionally dramatized events because we assimilate them as personal experiences. The never-ending, ever-morphing cyberspace narrative is a place to revel in a sense of endless 
transformations." This is why more people enjoy playing sports than watching them as spectators. More importantly, however, is that this is what actors do on stage when they bring a character to life. They enact a character.

We can play all the parts; exhaust all the possible outcomes, but as each enactment unfolds that becomes a linear story. Plots are composed of scenes that follow one upon the other. We can understand this more easily by recognizing each day of our lives as possessing its own particular plot by virtue of the events that transpired one upon the other. If we lived only one day, life would be defined by the story of that day. The events that unfolded in that particular order would define our story of life. We lament the passing of a young person because he or she has been denied the ability to live out/act out the myriad possibilities inherit in the game/the story of life.

Empowering the actor to make decisions that affect character interrelationships and story outcome (Murray's definition of agency) is connected implicitly to non-linearity. This is also the reason why sports and gaming are the best metaphors to capture the unique capabilities of digital media. A game is a kind of abstract storytelling that resembles the world of common experience, but compresses it in order to heighten interest. Every game, electronic or otherwise, can be experienced as a symbolic drama. Each move in a game is like a plot event in a simple but compelling story.

Let's use an example that both Japanese and American audiences can relate to easily: baseball. We know that baseball is a game featuring two teams, nine innings (most of the time), 27 outs (for the loser), three strikes for an out, four balls for a free pass to first base, etc., there is, in other words, a rule base within which myriad decisions and actions may be undertaken. The rules of this game are such that the odds of any two games unfolding in the exact same manner are considered rare. In fact, consistency is something not to be aspired to for fear of expectancy, redundancy and the negation of possibility. Winning all of the time becomes as tedious as losing all of the time. The sports debate raging in the United States currently is that baseball's financial structure favors the rich whereas football's salary cap engenders the hope that any team has a fair chance of winning the championship at the beginning of the year. In the U.S. we simply expect the Yankees to be in the World Series every year!

Non-linearity means therefore that the actor has the ability to make decisions affecting the actions and outcome of the game, story, and world. There are different types of plot structure, but most storytelling, at least in western cultures, is built upon a familiar, time-proven configuration: climactic plot structure. Climactic plot structure calls for an inciting incident, exposition or backstory, rising action, progressive complications, crisis, climax, and denouement. While the origins of this structure date back to 
Aristotle, it is entrenched firmly in western storytelling. This plot structure crosses myriad story genres: adventure, strategy, mystery, and most roleplaying. The human engine behind climactic plot structure is cause-andeffect reasoning. Climactic plot structure appeals to us because of its innate problem-solving dynamic, problem-solving being one of the ontological foundations of human existence. Interactive drama, however, lends itself more easily to episodic plot, where specific cause-and-effect reasoning takes a back seat to thematic development. We often say in theatre that climactic plot structure is the domain of the super-objective whereas episodic plot structure is the domain of the super-ego. The challenge becomes therefore: how do you bring episodic plot and climactic plot together?

Janet Murray makes an astute observation when she says; "We must realize that we cannot bring to a transformative, shape-shifting medium the same expectations of static shapeliness and finality that belong to linear media." The major gamble presented by digital entertainment is that by allowing any given actor to effect the outcome of the story, the risk is run that some of these played out scenarios will be, bluntly, boring.

Non-linearity also allows an actor to replay scenes by choice, or because the resulting actions led to finality (usually resulting in 'death' of the protagonist). This invitation to repeat actions, to relive episodes, to utter different statements, is made possible by digital technology. The experience, though, still unfolds as a linear plot.

While non-linearity does not mean that we need to forego a sense of completeness and emotional release, creating story and character options that adhere to the tents of climactic story structure is monumentally difficult. A digital playwright needs to be extremely clever to arrange dramatic action with such precision that it sustains suspense over multiple pathways. This is why Murray asks the extremely important question: "Could a digital narrative offer a higher degree of agency while still preserving the sense of tragic inevitability...can we construct a composite view of human action and interaction that does not resolve into any single story, but instead composes itself into a coherent system of interrelated actions?"

At this juncture, our answer has to be: we think so. It is hard to imagine, though, an imaginary world offering so many options for actor agency that each decision opens the door onto a climactic plotline. The long view of traditional storytelling reveals that soap operas (daytime or prime time) are structured in that manner. Each of the multiple plots is crafted as a climactic plot structure, affecting all of the characters inhabiting the world. The rub is that a non-linear playwright would have to craft all of these plots, subplots, tertiary plots, obstacles, etc., prior to opening the door to the outside actor. Another option would be a stable of writers who are crafting and producing 
extended story lines in accordance with the decision-making history of subscribers.

How then do we summarize or encapsulate non-linearity? We acknowledge actor empowerment as a dynamic effecting plot development. The ability of the actor (or "interactor" as Murray refers to him or her) to participate in the story means various stories will unfold and result. Nonlinearity is temporal in nature because it requires conscious and instinctive decision-making that then affects a world and the inhabitants of that world; non-linearity resides squarely in the world of cause-and-effect reasoning.

Most importantly though, non-linearity means you can play again. The virtual world, now more clearly than ever before, resembles real life, much more so than the stage or screen. That is the true nature of the digital entertainment revolution. Your actions now have genuine consequences, and you have the opportunity to reflect upon those actions and consequences and try to do better next time, or, at the very least, experiment. And experimentation is just another word for trying to figure out who you are. 
2. COMPUTERS \& GAMES 